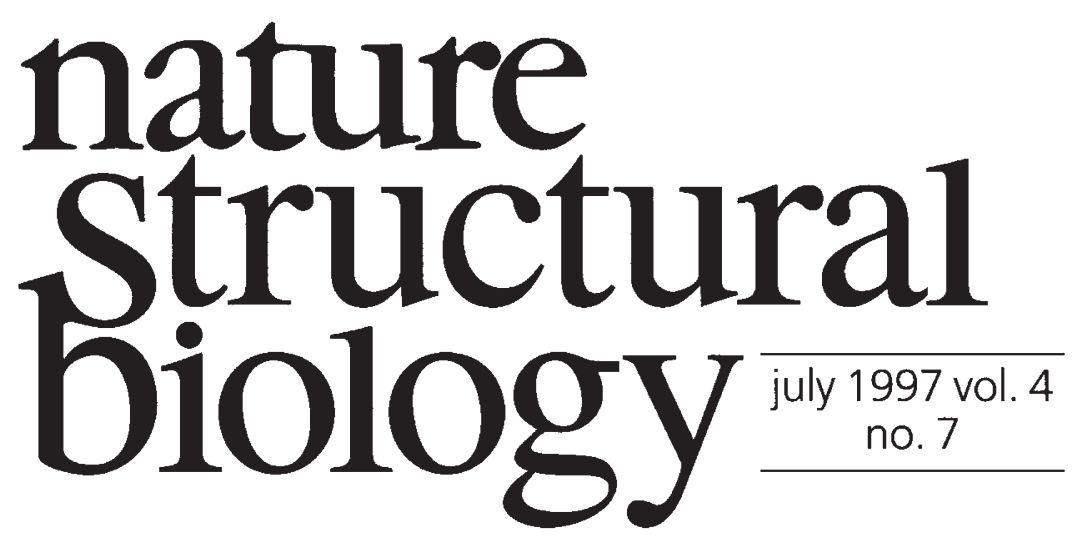

\title{
Nice splice
}

In hindsight, that clearest of all vision, it is perhaps unsurprising that a gene should be found coding for a protein-within-a-protein, and that the 'buried' polypeptide chain should be excised by protein (rather than RNA) splicing. After all, the chemistry involved is fairly mundane. And there have been upwards of 3.8 billion years for such exotic genetic and molecular phenomena to appear. What do these protein introns, or inteins, look like? Luckily, we are now treated to the first sight of the structure of an excised intein, PI-SceI from Saccharomyces cerevisiae, reported in a recently published paper

from Quiocho and colleagues 1 .

Inteins show some sequence similarities with members of the family of so-called homing endonucleases. This is a class of proteins found, for the most part, within group I or archeal self-splicing introns. Not all

IMAGE UNAVAILABLE FOR COPYRIGHT REASONS such introns contain endonucleases, but those that do have the ability to transfer themselves from an introncontaining $\left(\mathrm{I}^{+}\right)$to intronless $\left(\mathrm{I}^{-}\right)$allele of the same gene - a process known as intron homing ${ }^{2}$. Inteins are capable of the same feat: the sequence-specific doublestranded break catalyzed by the endonucleases and by inteins initiates the unidirectional gene conversion event that results in transfer. This ability allows them to promote their own dissemination. Indeed, homing would be expected to disperse group I introns and

Fig. 1 Structure of an intein. The nuclease domain (II) forms the upper part of the protein. Helices $\alpha 4$ and $\alpha 7$, which contain the dodecapeptide motif, are at the centre of the domain: the position of the putative active site Asp residues are shown in red. The third suggested active site residue, Lys 301 in the loop following $\beta 18$, is also indicated in red. The local two fold symmetry of the two domain-ll subdomains passes between the $\alpha 4$ and $\alpha 7$ helices and is perpendicular to the plane of the page. The intein splicing domain forms the lower, elongated part of the molecule and consists mostly of $\beta$-sheet. The splicing active site is on the back side of the molecule as shown, its position being marked by the $\mathrm{N}$ and $\mathrm{C}$ termini of the intein (reproduced with kind permission from ref. 1. Copyright Cell Press) inteins into all organisms possessing an appropriate recognition sequence.

Inteins differ from the homing endonucleases, though, in that they are synthesized as part of the amino acid sequence of another, completely unrelated protein and, presumably as a function of this most unusual location, they also have the ability to excise themselves from the precursor protein and then splice together the $\mathrm{N}$ - and C-terminal portions of the two ends of the 'protein exons' (or exteins) that contain them. Because of their intimate association with the exteins, retention (or precise deletion) of inteins would tend to be selected for, as any disruption of the protein splicing event would very likely inactivate the extein.

Colonization of a group I intron by an endonuclease benefits both entities. The intron, through its ability to splice itself out of the RNA transcript, provides a genetically silent haven for the invading endonuclease. The intron, on the other hand, gains mobility, a property that allows it to defy the forces of genetic streamlining (important in prokary- 
otes). Indeed, the persistence of these introns in modern organisms may be a direct result of their having acquired the ability to move within and between genomes ${ }^{3}$. Inteins combine these splicing and homing functions within a single molecule. Thus inteins, like the self-splicing group-1 introns, are a type of selfish genetic element or benign parasitic DNA.

There is some suggestion that inteins are not always phenotypically silent, though. The Rec A genes from both Mycobacterium leprae and M.turberculosis have independently acquired inteins, as indicated by the inteins' quite different size, sequence and location of insertion. The rarity of intein insertion suggests that the presence of inteins in these genes is not merely a matter of coincidence, rather it would seem that the inteins are serving some as yet unknown function in the host organisms ${ }^{4}$.

PI-SceI, the subject of Quiocho and colleagues attentions ${ }^{1}$, is sequestered within a subunit of the vacuolar ATPase (VMA1) of yeast. The protein splicing and endonuclease activities of the intein are independent of one another. Consistent with this, the structure of the protein consists of two separate domains, I and II, that house the splicing and nuclease activities respectively (Fig. 1).

The smaller domain, domain II, contains the putative nuclease active site. The domain also contains two dodecapeptide sequences that are a distinguishing characteristic of the homing endonucleases and inteins. In PI-SceI they form helices $\alpha 4$ and $\alpha 7$, at the $\mathrm{C}$-terminal ends of which are found two putative active-site Asp residues. They also form part of the 'interface' between the two structurally similar subdomains that constitute domain II. Similar observations have been noted in a paper reporting the first structure of a homing endonuclease, I-CreI ${ }^{5,6}$, where the dodecapeptide sequences play a role in dimer formation and catalysis. Clearly, conservation of these motifs is required for forming and maintaining the appropriate endonuclease active site geometry.

The active site of the intein splicing domain (domain $\mathrm{I}$ ) — formed around the $\mathrm{N}$ and $\mathrm{C}$ termini of the protein - is on the opposite side of the molecule from the nuclease active site. Protein splicing is an autocatalytic event, the major determinants of which been shown to reside within the intein and the first residue of the $\mathrm{C}$-terminal extein. A hydroxyl- or thiol-containing residue (Ser, Thr or Cys) is always present at the positions that immediately follow the two splice junctions (Cys 1 of PI-SceI and Cys 455 of the Cterminal extein) and the sequence His-Asn (His 453-Asn 454) is invariant at the intein C terminus.

Mutagenesis and biochemical analysis has revealed the reaction pathway for splicing: (i) an $\mathrm{N}-\mathrm{S}$ acyl rearrangement involving Cys 1 , converting the peptide bond at the $\mathrm{N}$ extein-intein junction into a thiolester linkage; (ii) transesterification, occurring through nucleophilic attack of the $\mathrm{N}$-terminal junction by Cys 455 , resulting in a branched intermediate; (iii) peptide cleavage, releasing the intein from the branched intermediate, coupled to succinimide formation involving Asn 454; and (iv) an S-N acyl shift, converting the extein-extein thioester link into a peptide bond. All these residues are found to be in close proximity to one another in a cavity in domain $\mathrm{I}$ : the $\mathrm{N}$ and C termini, Cys 1 and Asn 454, are as little as $2.9 \AA$ apart; the invariant His 79 is close to Cys 1; and the side chain of His 453 is in close proximity to Asn 454 . Of course, details of the conformation of the active site may be somewhat different in the precursor protein.

Duan, X., Gimble, F.S. \& Quiocho, F.A. Cell 89 , 555-564 (1997).

2. Lambowitz, A.M. \& Belfort, M. Ann. Rev Biochem. 62, 587-622 (1993).

3. Loizos, N., Tillier, E.R.M. \& Beifort, M. Proc. Natl. Acad. Sci. USA 91, 11983-11987 (1994).

4. Davis, E.O., Thangataj, H.S., Brooks, P.C. \& Colston, M.J. EMBO / 13, 699-703 (1994).

5. Heath, P.J., Stephens, K.M., Monnat Jr., R.J. \& Stoddard, B.L. Nature Struct. Biol. 4, 468-476 (1997)

6. Aggarwal, A.K. Nature Struct. Biol. 4, 423-424 (1997).
Quiocho and colleagues speculate that the intein arose by the invasion of the endonuclease into a gene encoding for a protein that had, or later developed splicing activity. The structure is certainly suggestive of this; domain II looks as if it is embedded within the middle of the protein splicing domain I (Fig. 1). The pairing of endonuclease and splicing function, exactly analogous to the group-1 homing intron, defines a minimal mobile genetic unit. One wonders what other bizarre molecular beasts are waiting to be found. 